Supplemental Material for

\title{
Five New Zinc Phosphite Structures: Tertiary Building Blocks in the Construction of Hybrid Materials
}

Jian Fan, Carla Slebodnick, Diego Troya, Ross Angel, Brian E. Hanson* 


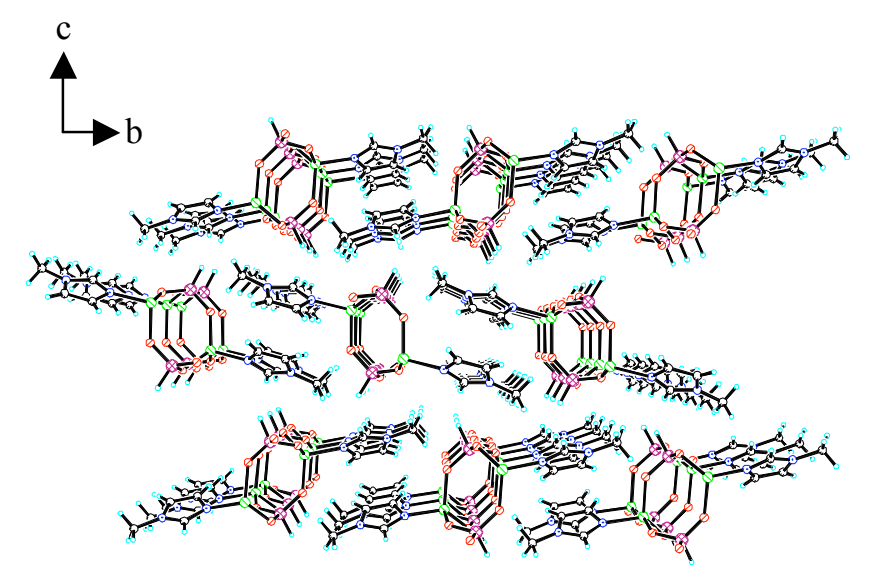

Figure S1. Cr:
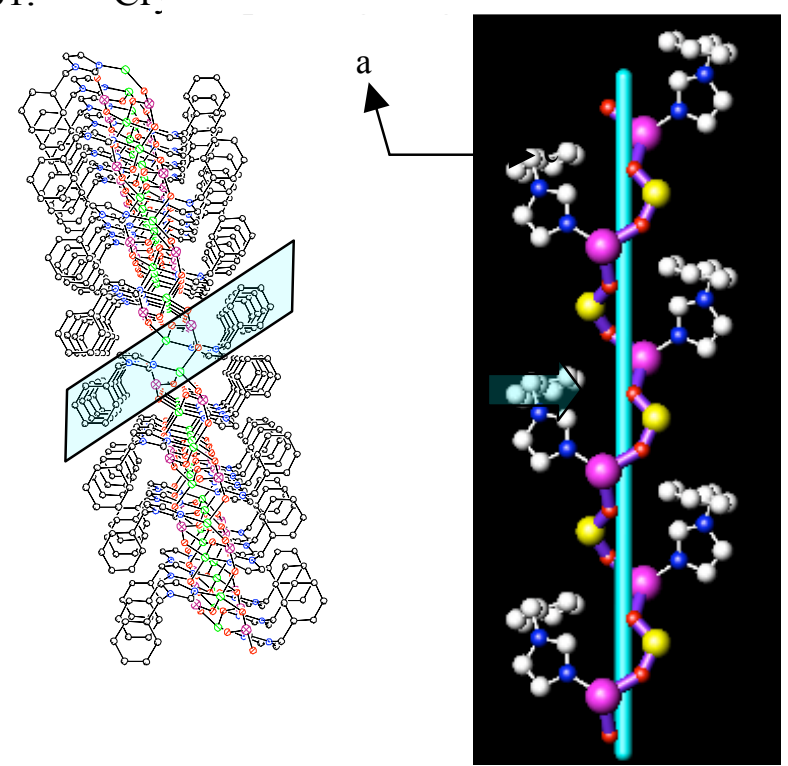

Figure S2. Side view of the 2D structure in $\mathbf{2}$ with the helical motif highlighted within blue square (left) and the helix (right, P: yellow, Zn: pink, O: red, N: blue and C: white).

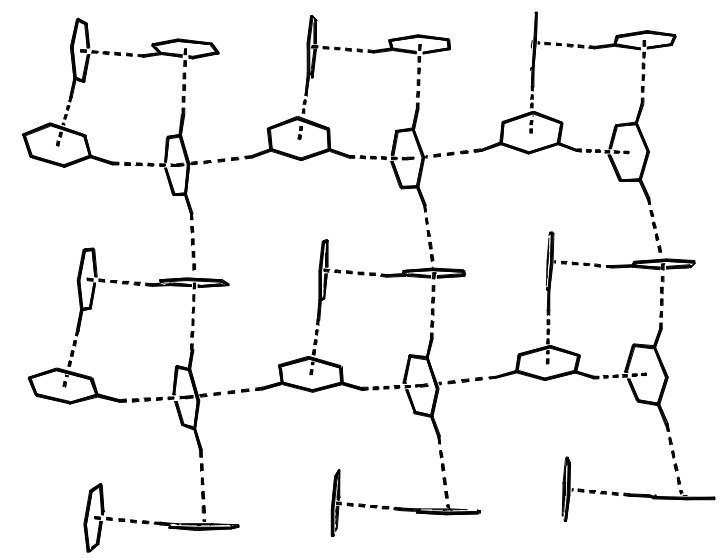

Figure S3. View of the arrangement of benzene rings in compound 2 along [001] direction with $\mathrm{C}-\mathrm{H} \cdots \pi$ interaction indicated by dashed lines. 


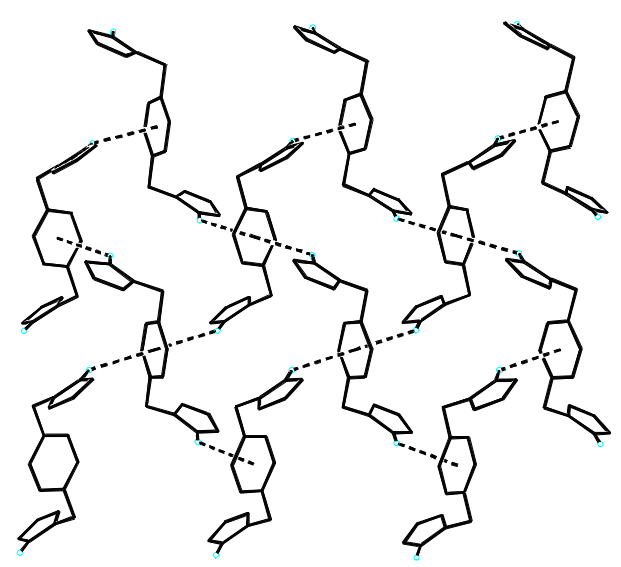

Figure S4. View of the arrangement of organic ligands in compound 3 with $\mathrm{C}-\mathrm{H} \cdots \pi$ interaction indicated by dashed lines.

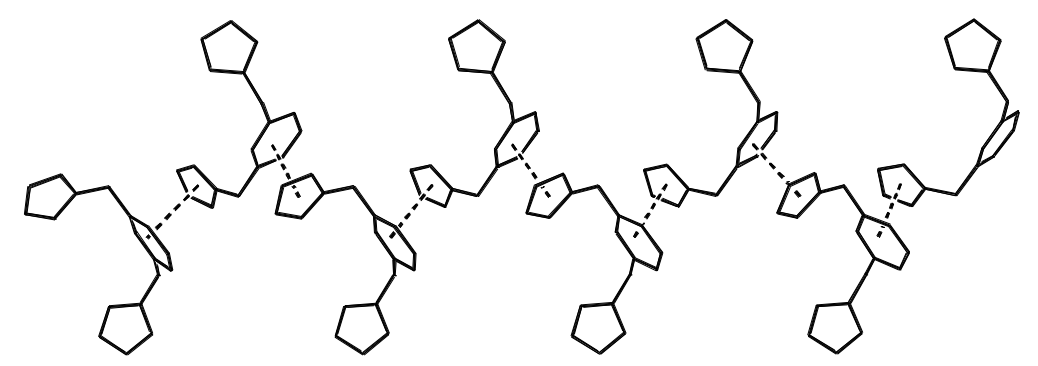

Figure S5. View of the arrangement of organic ligand in compound 4 along [001] direction with $\pi \cdots \pi$ interaction indicated by dashed lines. 


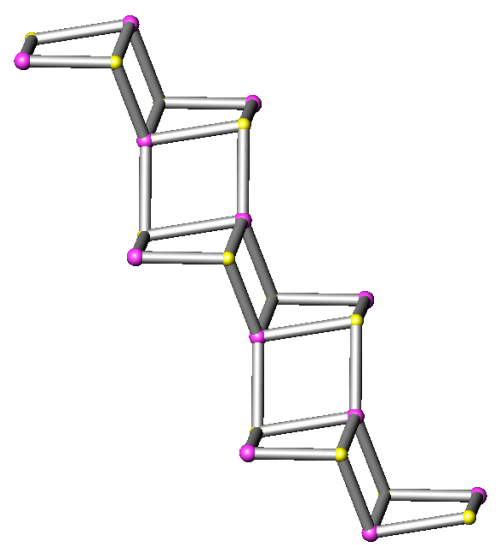

Figure S6. View of the 1D inorganic chain in 4 in a stair-step arrangement. 


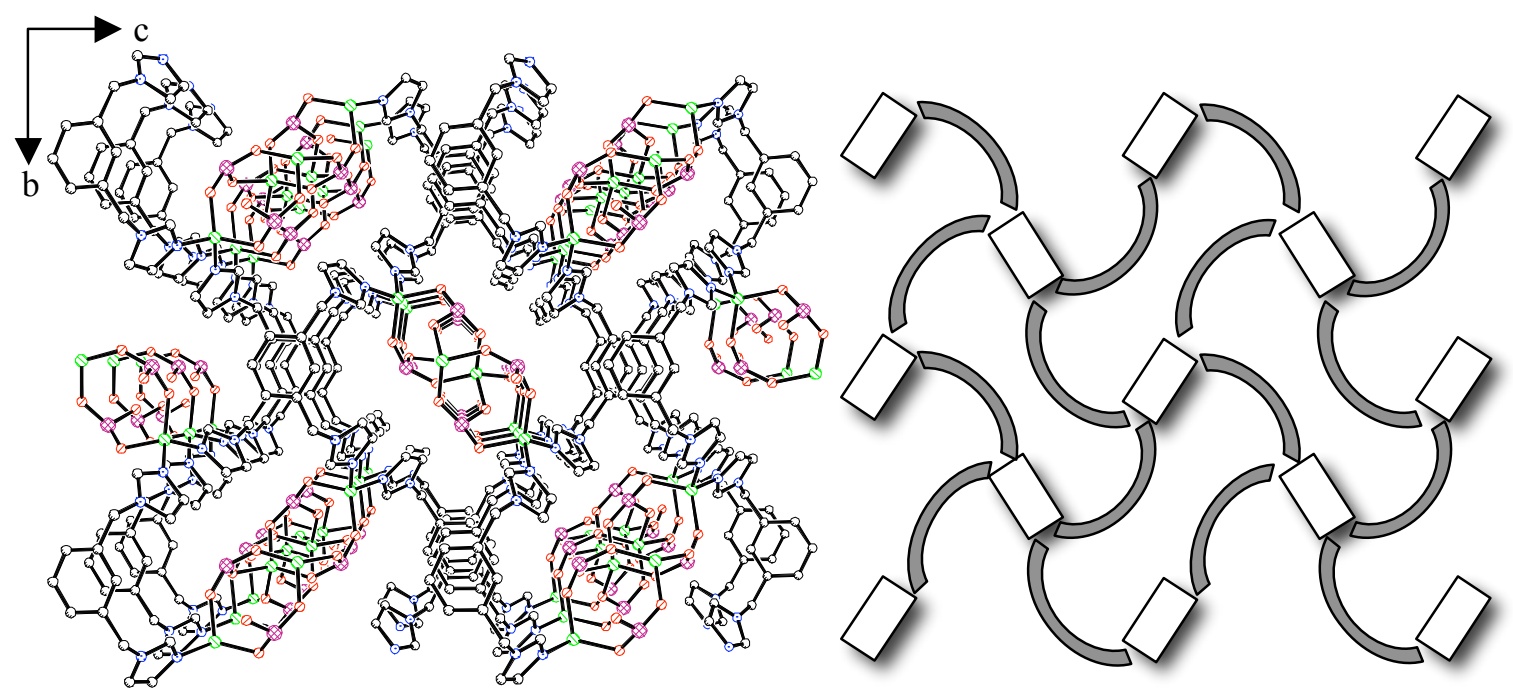

Figure S7. Crystal packing diagram for compound 4 in the $b c$ plane with hydrogen atoms omitted for clarity (left) and a schematic rendering of the connection pattern between 1D inorganic chains (rectangle) and the organic ligand (curve) in the $b c$ plane. 